Article

\title{
Immunohistochemical Expression Patterns of Tight Junction Proteins, Pro-Apoptotic and Anti-Apoptotic Factors on Progression of Intestinal Mucositis of Onco-Hematological Patients under Epirubicin-Based Chemotherapy
}

\author{
Coralia Cotoraci $^{1, *, \dagger}$, Alciona Sasu ${ }^{1, \dagger}$, Eftimie Miutescu ${ }^{2, \dagger}$, Dana Iovanescu ${ }^{2}$, Sami Gharbia ${ }^{3, \dagger}$, Alina Ciceu ${ }^{3}$, \\ Hildegard Herman ${ }^{3}(\mathbb{D})$ and Anca Hermenean ${ }^{3,4, *}$ \\ 1 Department of Hematology, Faculty of Medicine, Vasile Goldis Western University of Arad, 310025 Arad, \\ Romania; sasu.alciona@uvvg.ro \\ 2 Department of Gastroenterology, Faculty of Medicine, Vasile Goldis Western University of Arad, 310025 Arad, \\ Romania; miutescu.eftimie@uvvg.ro (E.M.); iovanescu.dana@uvvg.ro (D.I.) \\ 3 "Aurel Ardelean" Institute of Life Sciences, Vasile Goldis Western University of Arad, 310025 Arad, Romania; \\ gharbia.sami@uvvg.ro (S.G.); ciceu.alina@uvvg.ro (A.C.); hildegard.i.herman@gmail.com (H.H.) \\ 4 Department of Histology, Faculty of Medicine, Vasile Goldis Western University of Arad, \\ 310025 Arad, Romania \\ check for \\ updates \\ * Correspondence: cotoraci.coralia@uvvg.ro (C.C.); hermenean.anca@uvvg.ro (A.H.) \\ + These authors contributed equally to this work.
}

Citation: Cotoraci, C.; Sasu, A.; Miutescu, E.; Iovanescu, D.; Gharbia, S.; Ciceu, A.; Herman, H.; Hermenean, A. Immunohistochemical Expression Patterns of Tight Junction Proteins, Pro-Apoptotic and Anti-Apoptotic Factors on Progression of Intestinal Mucositis of Onco-Hematological Patients under Epirubicin-Based Chemotherapy. Appl. Sci. 2021, 11, 4710. https://doi.org/10.3390/ app11104710

Academic Editor: Francisco Arrebola

Received: 18 March 2021

Accepted: 10 May 2021

Published: 20 May 2021

Publisher's Note: MDPI stays neutral with regard to jurisdictional claims in published maps and institutional affiliations.

Copyright: () 2021 by the authors. Licensee MDPI, Basel, Switzerland. This article is an open access article distributed under the terms and conditions of the Creative Commons Attribution (CC BY) license (https:// creativecommons.org/licenses/by/ $4.0 /)$.

Abstract: Chemotherapy and radiation are often accompanied by complications such as intestinal mucositis. The aim of this study was to assess by immunohistochemical assay the consequences of epirubicin-based therapy applied to onco-hematological patients, on the mucosal cells that undergo apoptosis and on the tight junction proteins, immediately before and after a short time of chemotherapy administration. We assessed the protein expression and distribution of the pro-apoptotic Bax, anti-apoptotic $\mathrm{Bcl}-2$ and effector Caspase-3 as key proteins in apoptosis pathways and the changes in immunopositivity of Claudin-1 and ZO-1 tight junction proteins. Results show that the Bcl-2 family is involved in intestinal damage via Caspase-3 dependent apoptosis of epithelial cells. Additionally, the intestinal mucositis activates other injurious pathways through a dramatic drop in Claudin- 1 and ZO-1 expressions, contributing for a while to a structural and functional integrity disruption of the intestinal epithelium.

Keywords: onco-hematological patients; epirubicin; colon; apoptosis; tight junction

\section{Introduction}

Chemotherapy and radiation are often accompanied by complications such as intestinal mucositis. This is represented by the disturbance of the intestinal mucosa integrity and is clinically manifested by diarrhea, painful inflammation and ulceration, and in severe cases, hemorrhage, sepsis, ischemia and the formation of strictures [1]. This seems to reflect the high sensitivity of the gastrointestinal tract epithelium to cytotoxic therapies due to the high turnover rate of these cells [2]. Injury to the intestinal epithelium leads to disruption of the integrity of the surface mucosal barrier and allows changes in permeability to various substances and the translocation of bacterial products from the lumen, which contributes to morbidity and mortality. Therefore, gastrointestinal toxicity frequently limits the use of radiation and chemotherapy for the treatment of patients with a wide variety of malignancies [2].

The maintenance of intestinal homeostasis is closely related to the integrity of the epithelial layer renewal. The intestinal epithelial monolayer is composed of different types of specialized epithelial and goblet cells, each with a distinct function. Goblet cells secrete mucous and resistin-like molecule- $\beta$, which are necessary for the defense 
and repair of the epithelial layer and in maintaining epithelial homeostasis [3,4]. This structural and functional integrity allows the permeability of essential ions and water but restricts the entry of pathogens and bacterial toxins [5]. The transport of molecules across the epithelial layer occurs through passive diffusion, the carrier-mediated pathway and the paracellular pathway between the spaces of adjacent cells. The barrier function of the epithelium is provided by the epithelial tight junction proteins, which seals the paracellular space between the apical part of two consecutive epithelial cells and restricts the transport of hydrophilic molecules [6-9]. The tight junction is composed of several proteins, including occludens; claudins; zonula occludens (ZO); and cingulin, tricellulin and junctional adhesion molecules, which have a complex network between them, as well as with the cytoskeleton. These proteins have specific roles: occludens provide structural integrity to the tight junction [10], claudins are responsible for the regulation of paracellular space [11] and ZO are essential for the connection of other tight junction proteins to the cytoskeleton $[12,13]$. Any alteration in the tight junction structure can be detrimental to the structural and functional integrity of the intestinal barrier.

Apoptosis plays an important role in determining the architecture of the intestinal epithelium and is also part of the stress response triggered by the toxicant. It appears as a single process, being triggered on several pathways which depend on the position of the cell along the crypt, its level of differentiation and the type of stimulus involved [14]. Under normal conditions, the integrity and normal functioning of the gut is ensured by the apoptosis rate of the gastrointestinal epithelial cells paired with the rate of mitosis [15]. In a previous study, we induced epirubicin-based mucosal barrier injuries of the gastrointestinal tract in mice and found that the small intestine is much more susceptible to damage-induced apoptosis by the intrinsic pathway, followed by the colon and stomach [16], but up to date, there have not been any patho-clinical observations of intestinal changes that follow a standard antineoplastic epirubicin-based protocol applied to onco-hematological patients.

The aim of this study was to assess by immunohistochemical assay the consequences of epirubicin-based therapy applied to onco-hematological patients, on the mucosal cells that undergo apoptosis and on the tight junctions' proteins, immediately before and after a short time of chemotherapy administration. This immunohistochemical analysis may be important for clinicians in correlating clinical signs with the molecular causes that led to the temporary disruption of the intestinal barrier and in determining when the intestinal epithelium begins to recover, which may be of interest for further therapeutic protocols.

\section{Materials and Methods}

\subsection{Oncohematological Patients-Clinical Evaluation and Colonic Biopsies}

The study was performed in the Hematology Clinic of the Arad Clinical Emergency County Hospital on 30 onco-hematological patients subject to 182 applications of standard antineoplastic epirubicin-based protocol. The experimental protocol was approved by the Bioethics Commission of the hospital. All data were collected between June 2015 and September 2016. Of the onco-hematological patients, 9 were biopsied after cumulating $6-8$ cycles of chemotherapy, $48 \mathrm{~h}$ and 3 weeks after the last epirubicin administration. The control was represented by healthy volunteers.

\subsection{Immunohistochemistry}

Five $\mu \mathrm{m}$ paraffin sections of colon biopsies were cut, dewaxed in xylene and rehydrated through a graded series of alcohols. Slides were washed $2 \times 5 \mathrm{~min}$ in PBS prior to being subjected to heat-mediated antigen retrieval in citrate buffer $(\mathrm{pH}$ 6.5). The endogenous peroxidase activity was blocked and slides were incubated overnight at $4{ }^{\circ} \mathrm{C}$ with the primary antibodies: rabbit polyclonal ZO-1 (ab214228), mouse monoclonal Bax (ab 5714), mouse monoclonal Bcl-2 (ab692), mouse monoclonal Claudin-1 (sc-166338) and mouse monoclonal Caspase-3 (sc-271759) (1:100 dilution) on the experimental section, and PBS only on the negative section. Immunoreactions were visualized employing a Novocastra Peroxidase/DAB kit (Leica Biosystems, Nussloch, Germany) according to the 
manufacturer's instructions. The protein expression of Bcl-2, Bax, Caspase-3, ZO-1 and Claudin-1 was quantified by the percentage of positive cells in 5 random fields by using a multi-functional cell image analysis software (cellSens Dimension, Olympus Life Science Solutions), thoroughly analyzing the histological section with a $20 \times$ objective.

\subsection{Statistical Data Analysis}

Statistical analysis was conducted with 1-way ANOVA using Prism software (GraphPad Inc., La Jolla, CA, USA) followed by Tukey's Multiple Comparison Test. A value of $p<0.05$ was considered to be statistically significant.

\section{Results}

\subsection{Effect of Epirubicin-Based Chemotherapy on the Expression of Tight Junction Proteins}

Maintaining the integrity of the intestinal barrier and the permeability of the intestinal epithelium is dependent on the structure of the tight junction proteins, as seen in $\mathrm{ZO}-1$ and Claudin-1. ZO-1 was well expressed in control slides (Figure 1) and reduced in both of the sigmoid segments from the onco-hematological patients $48 \mathrm{~h}$ following epirubicin-based chemotherapy (Figure 2). After 3 weeks of chemotherapy cessation, the expression of ZO-1 remained below the control (Figure 3). Claudin-1 was immunopositive in control slides (Figure 4 ) and significantly reduced $48 \mathrm{~h}$ after epirubicin administration (Figure 5) whereas after 3 weeks was almost restored (Figure 6). The weakest immunopositivity was recorded for ZO-1 under epirubicin treatment.
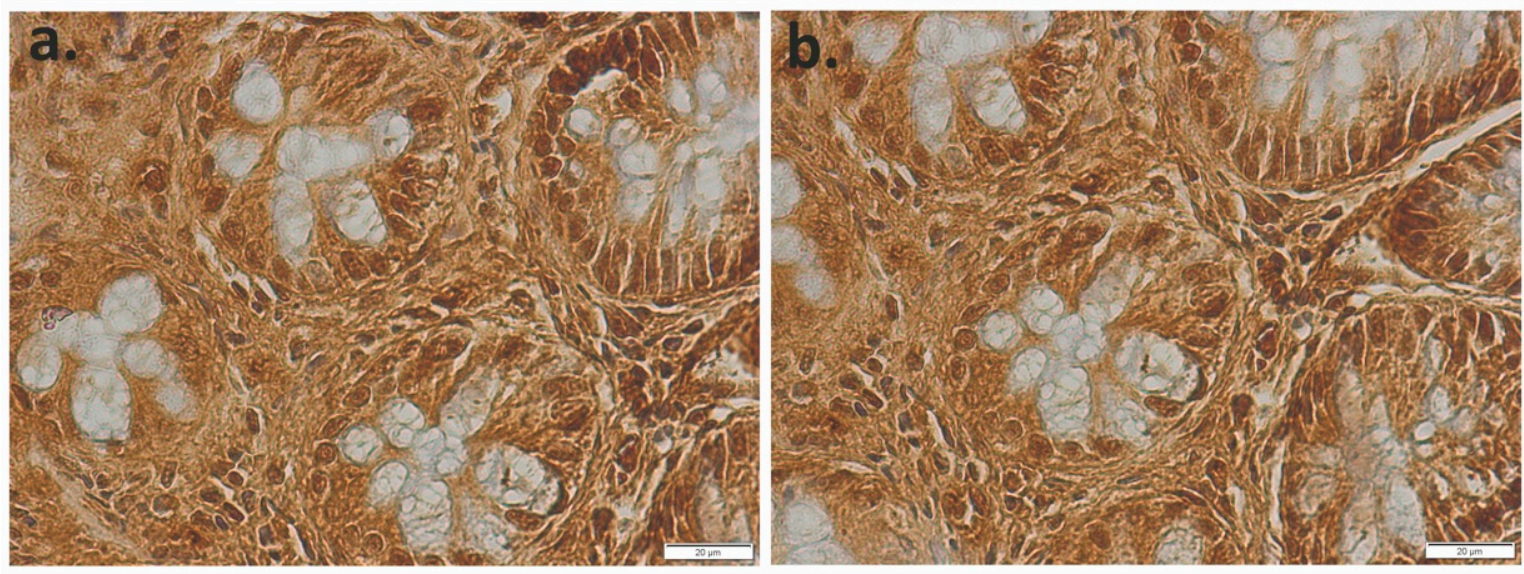

Figure 1. ZO-1 immunostaining in the proximal (a) and distal (b) sigmoid colon of the controls.
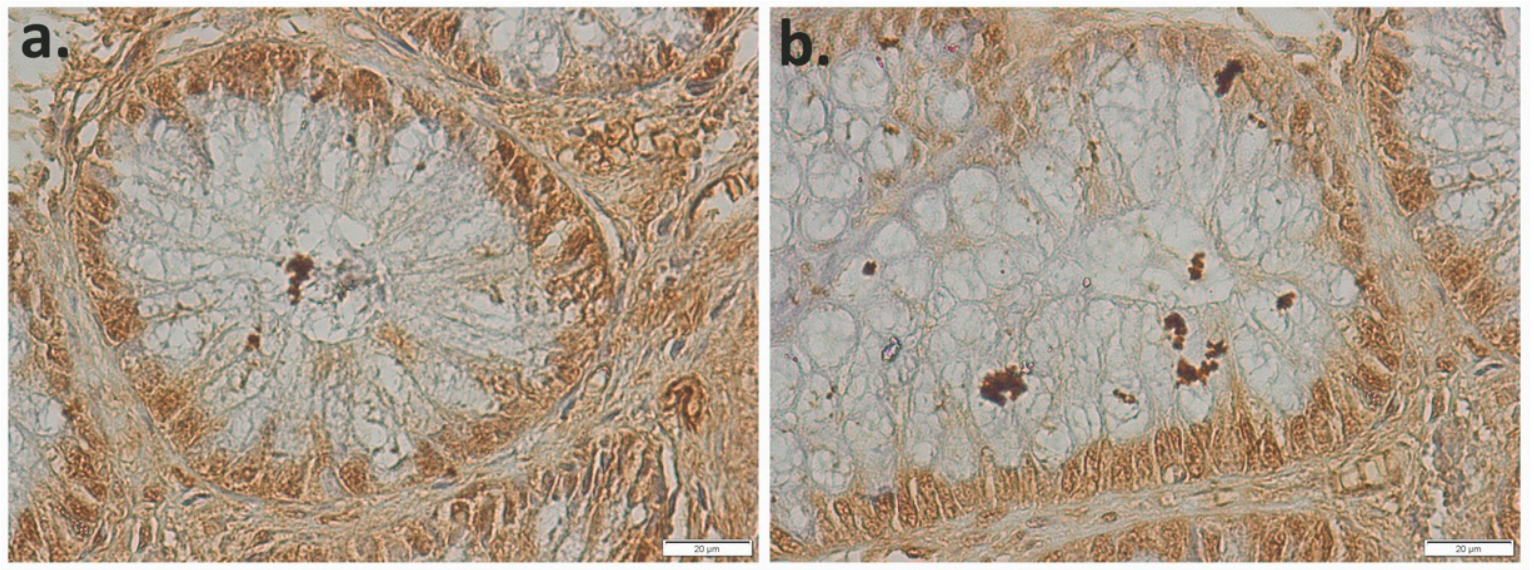

Figure 2. ZO-1 immunostaining in the proximal (a) and distal (b) sigmoid colon of the onco-hematological patients $48 \mathrm{~h}$ following epirubicin-based chemotherapy. 

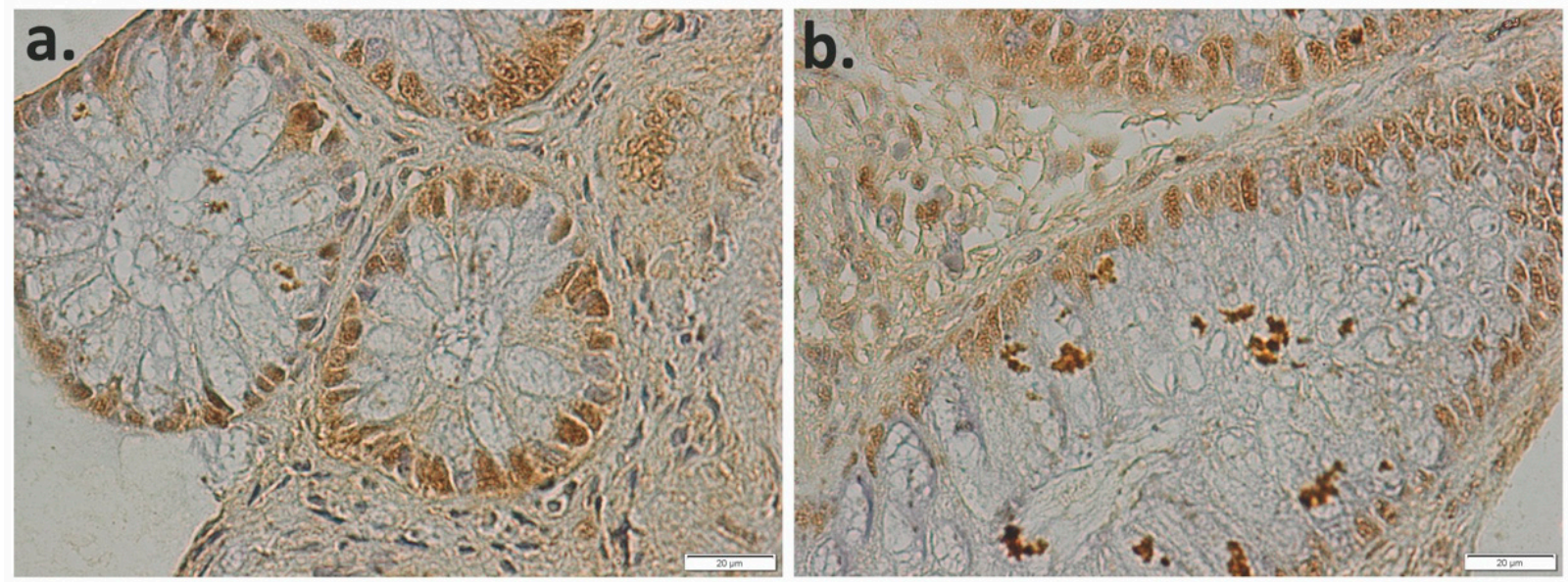

Figure 3. ZO-1 immunostaining in the proximal (a) and distal (b) sigmoid colon of the onco-hematological patients 3 weeks following epirubicin-based chemotherapy.
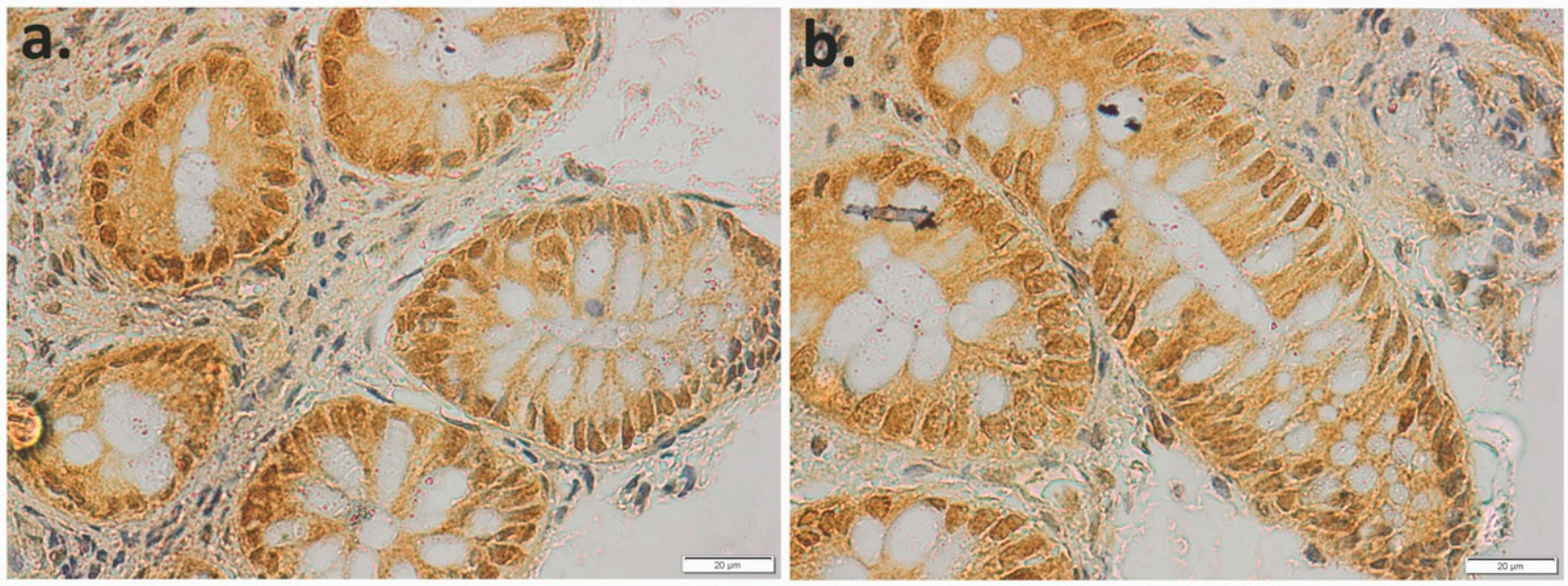

Figure 4. Claudin-1 immunostaining in the proximal (a) and distal (b) sigmoid colon of the controls.
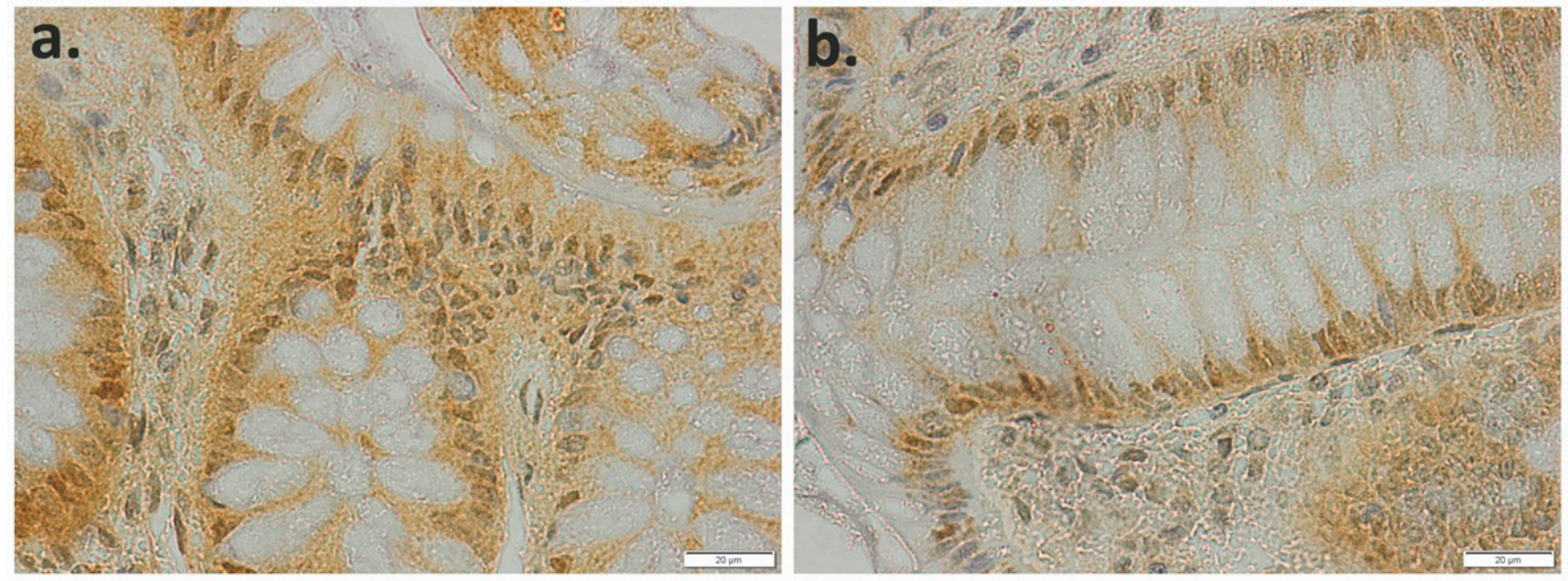

Figure 5. Claudin-1 immunostaining in the proximal (a) and distal (b) sigmoid colon of the onco-hematological patients $48 \mathrm{~h}$ following epirubicin-based chemotherapy. 

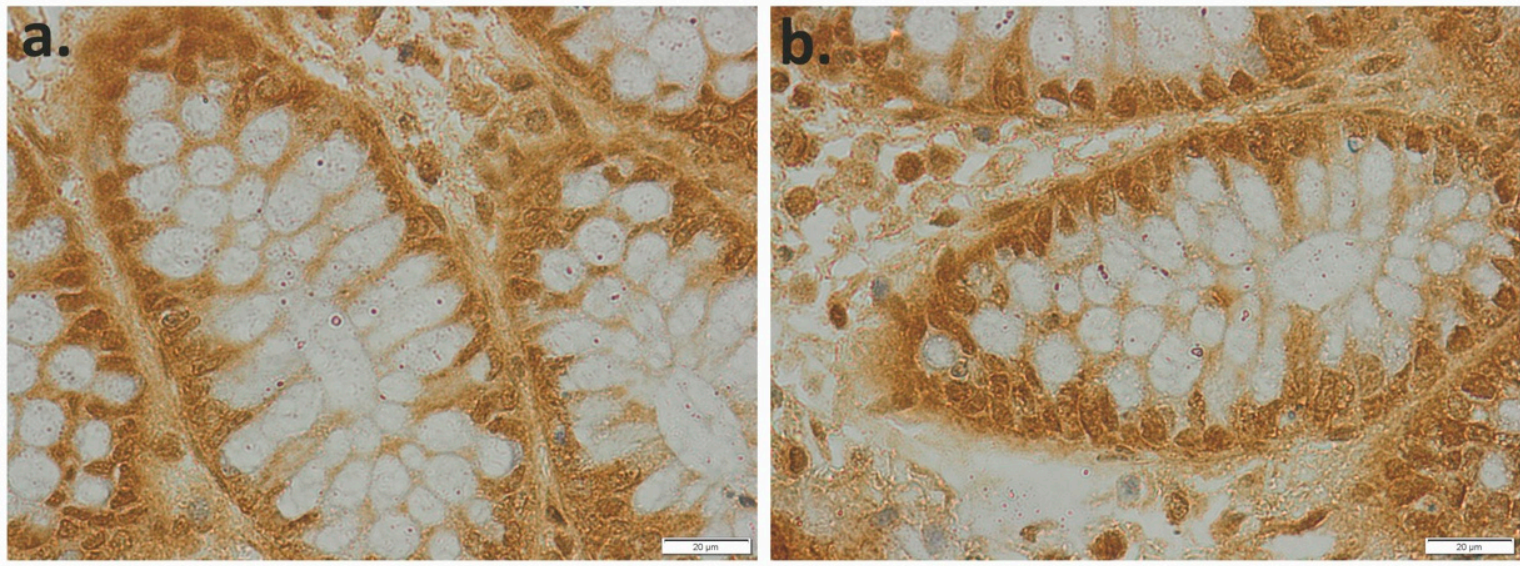

Figure 6. Claudin-1 immunostaining in the proximal (a) and distal (b) sigmoid colon of the onco-hematological patients 3 weeks following epirubicin-based chemotherapy.

\subsection{Effect of Epirubicin-Based Chemotherapy on the Expression of Apoptosis Markers}

The pro-apoptotic Bax immunostaining was negative in control (Figure 7) and markedly expressed $48 \mathrm{~h}$ following epirubicin-based chemotherapy (Figure 8). After 3 weeks of chemotherapy cessation, the expression of Bax was slightly reduced but still over the control (Figure 9). Similar, caspase-3 was negative in control (Figure 10) and significantly immunopositive at $48 \mathrm{~h}$ (Figure 11), whereas staining was decrease 3 weeks after chemotherapy (Figure 12).
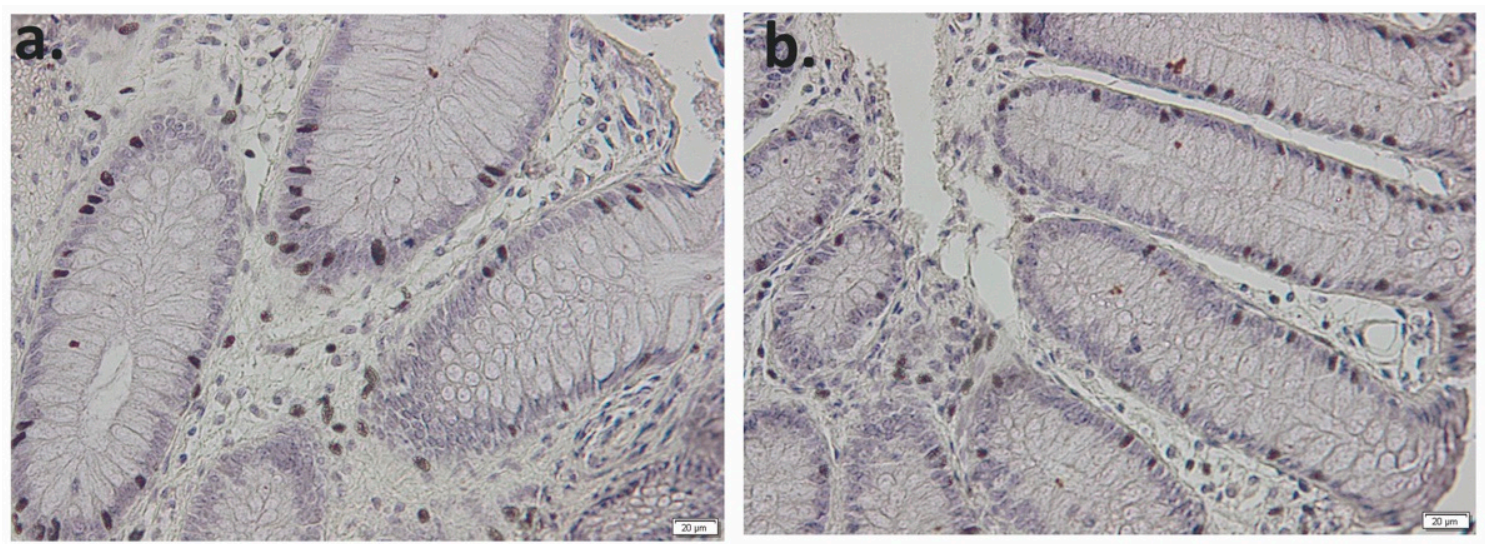

Figure 7. Bax immunostaining in the proximal (a) and distal (b) sigmoid colon of the controls.
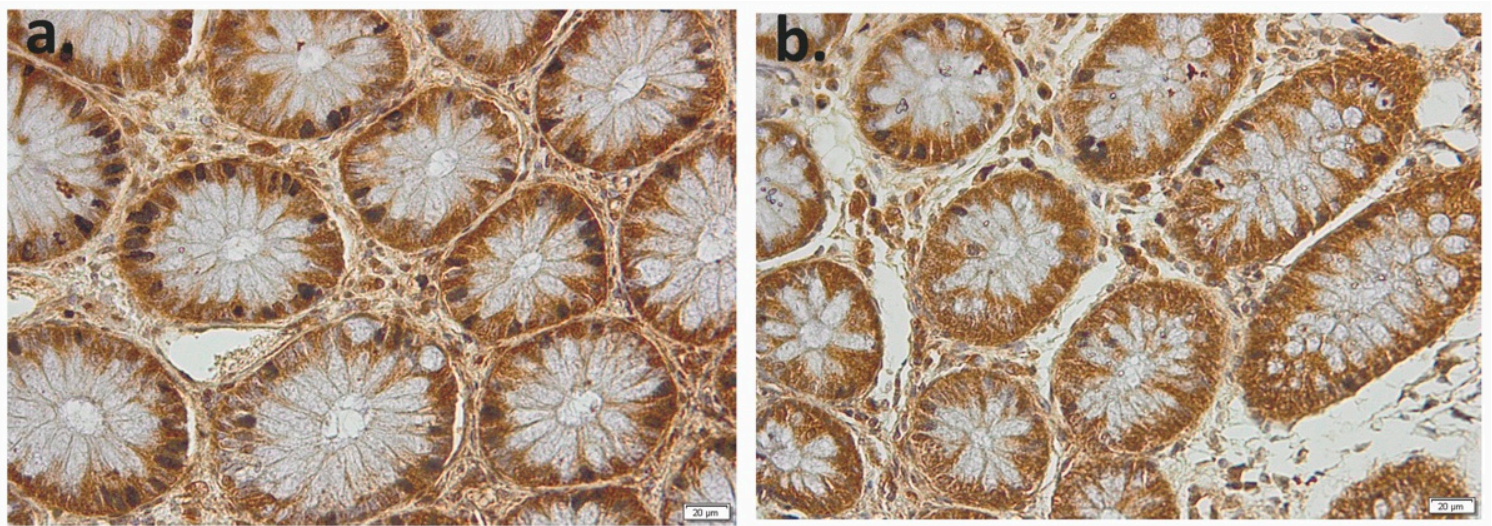

Figure 8. Bax immunostaining in the proximal (a) and distal (b) sigmoid colon of the onco-hematological patients $48 \mathrm{~h}$ following epirubicin-based chemotherapy. 

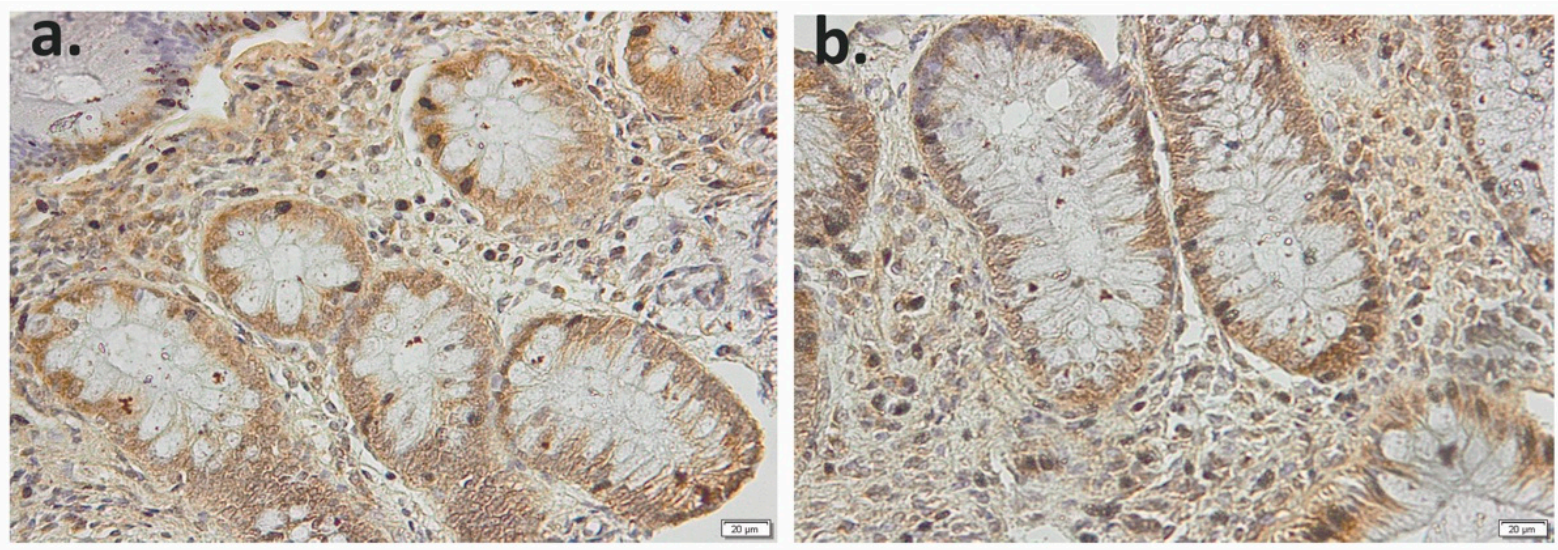

Figure 9. Bax immunostaining in the proximal (a) and distal (b) sigmoid colon of the onco-hematological patients 3 weeks following epirubicin-based chemotherapy.
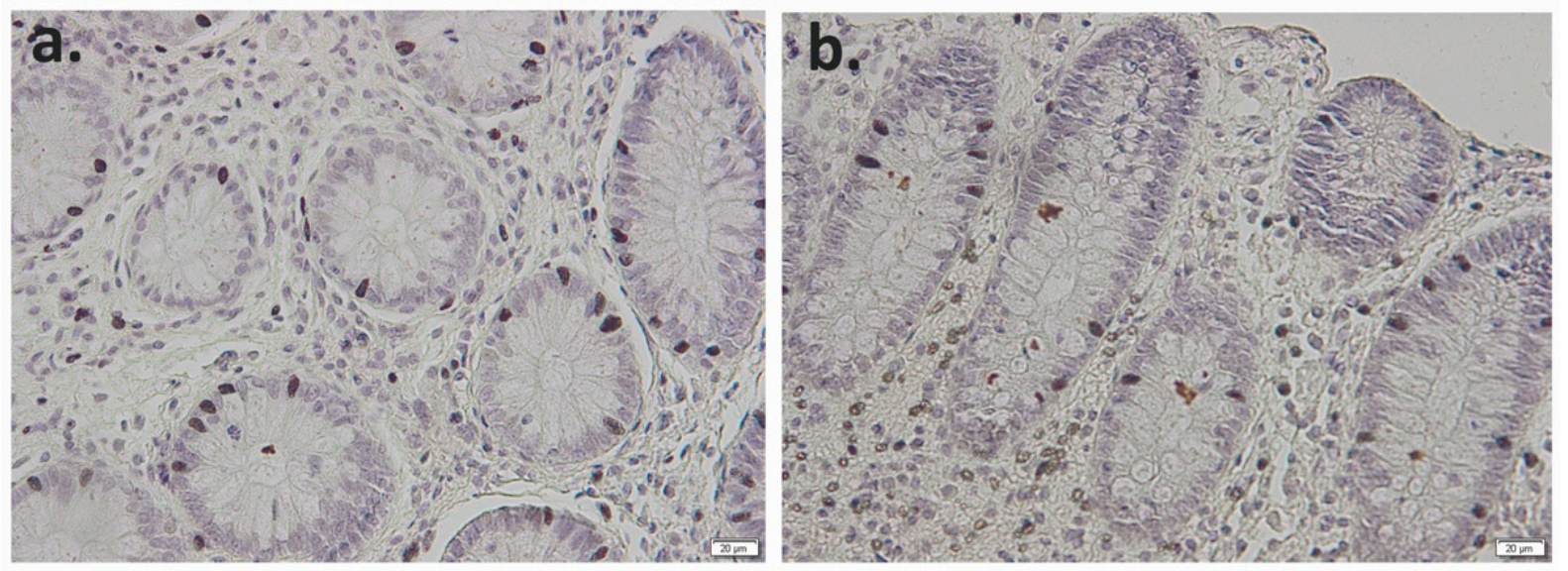

Figure 10. Caspase-3 immunostaining in the proximal (a) and distal (b) sigmoid colon of the controls.
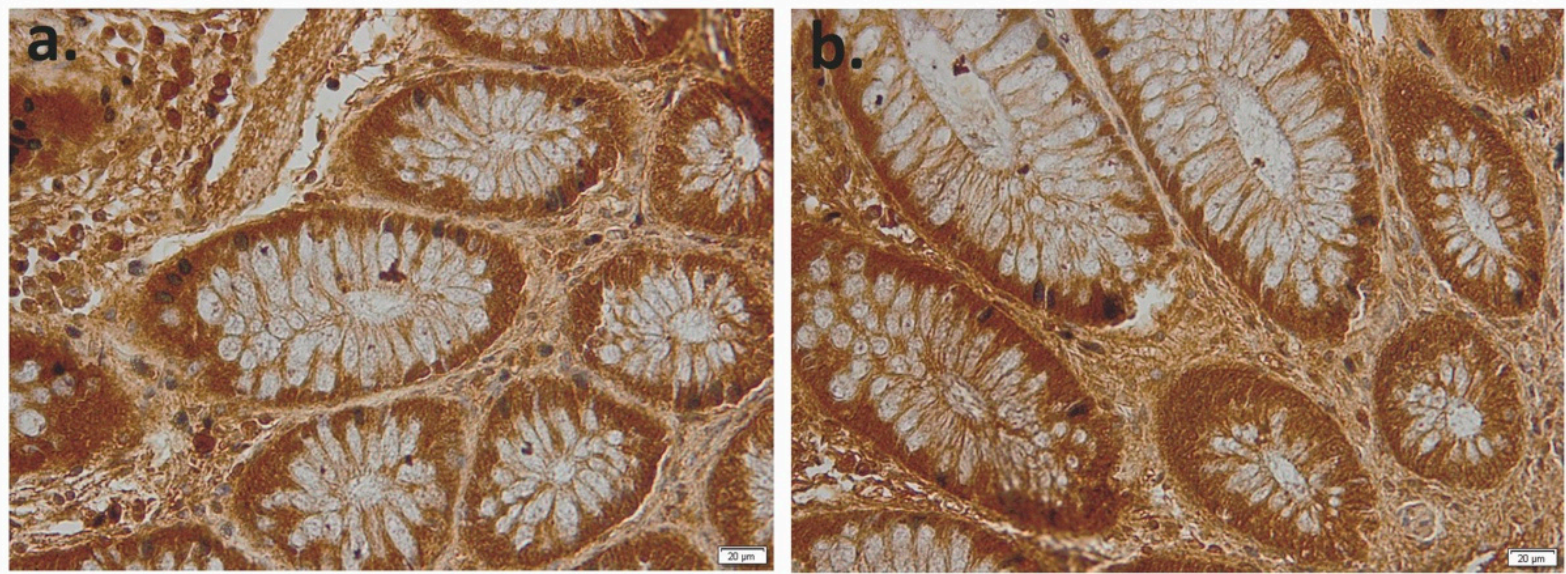

Figure 11. Caspase-3 immunostaining in the proximal (a) and distal (b) sigmoid colon of the onco-hematological patients $48 \mathrm{~h}$ following epirubicin-based chemotherapy. 

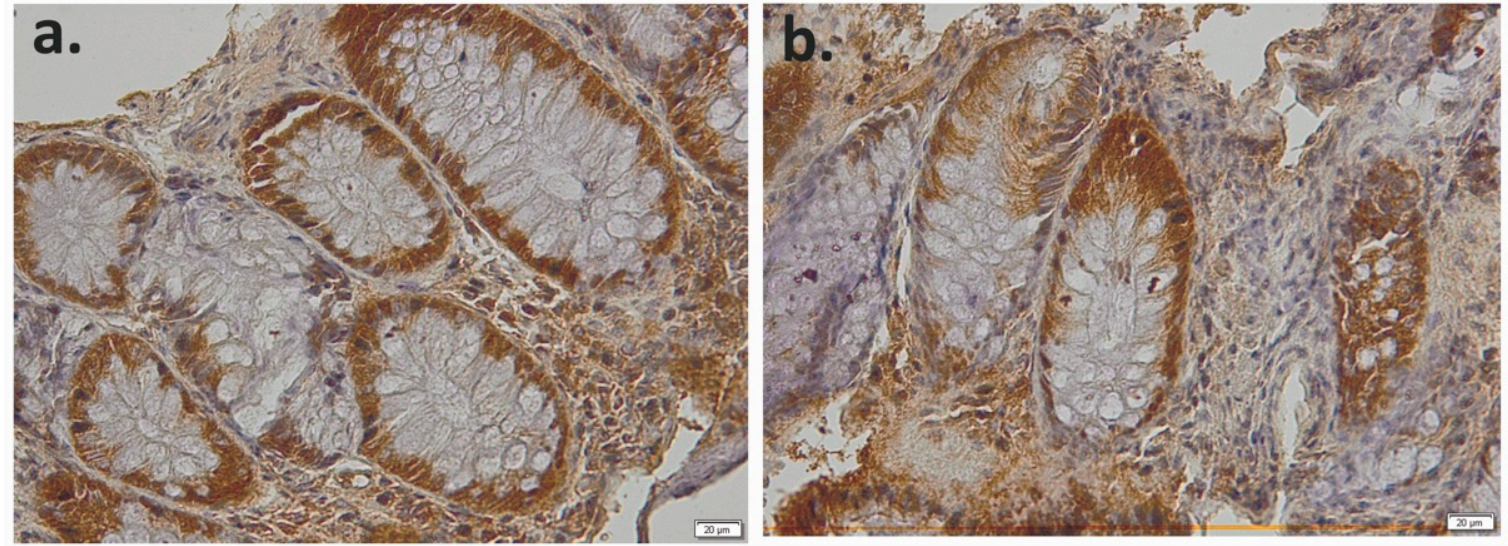

Figure 12. Caspase-3 immunostaining in the proximal (a) and distal (b) sigmoid colon of the onco-hematological patients 3 weeks following epirubicin-based chemotherapy.

On the contrary, the anti-apoptotic marker Bcl-2 was immunopositive in control (Figure 13), decreased $48 \mathrm{~h}$ following epirubicin-based chemotherapy, being present only in the chorion cells (Figure 14). After 3 weeks of chemotherapy cessation, the expression of Bcl-2 is still weak compared to control (Figure 15).
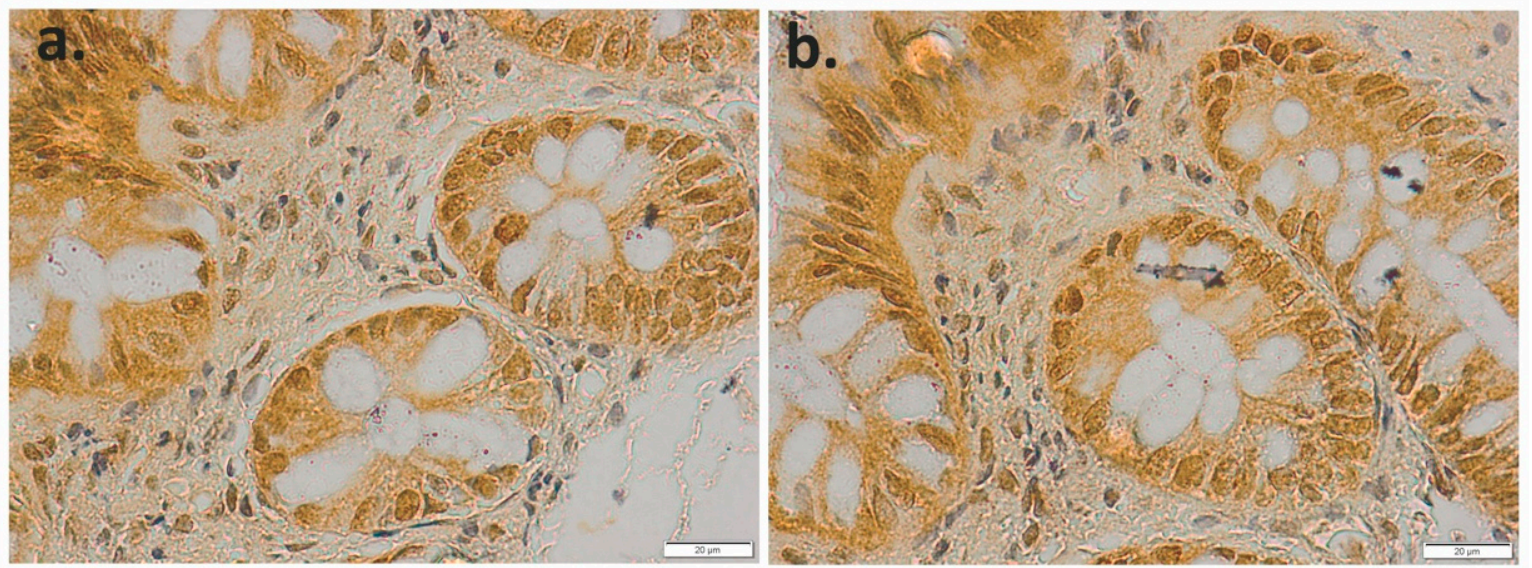

Figure 13. Bcl-2 immunostaining in the proximal (a) and distal (b) sigmoid colon of the controls.
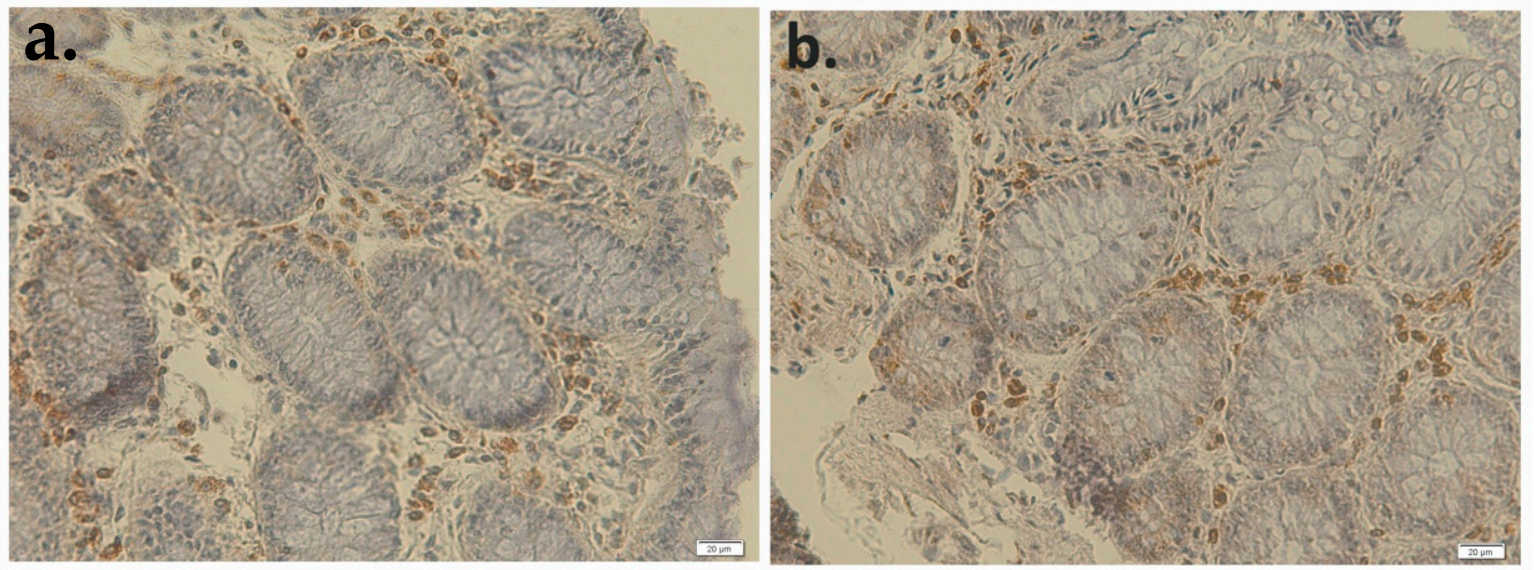

Figure 14. Bcl-2 immunostaining in the proximal (a) and distal (b) sigmoid colon of the onco-hematological patients $48 \mathrm{~h}$ following epirubicin-based chemotherapy. 

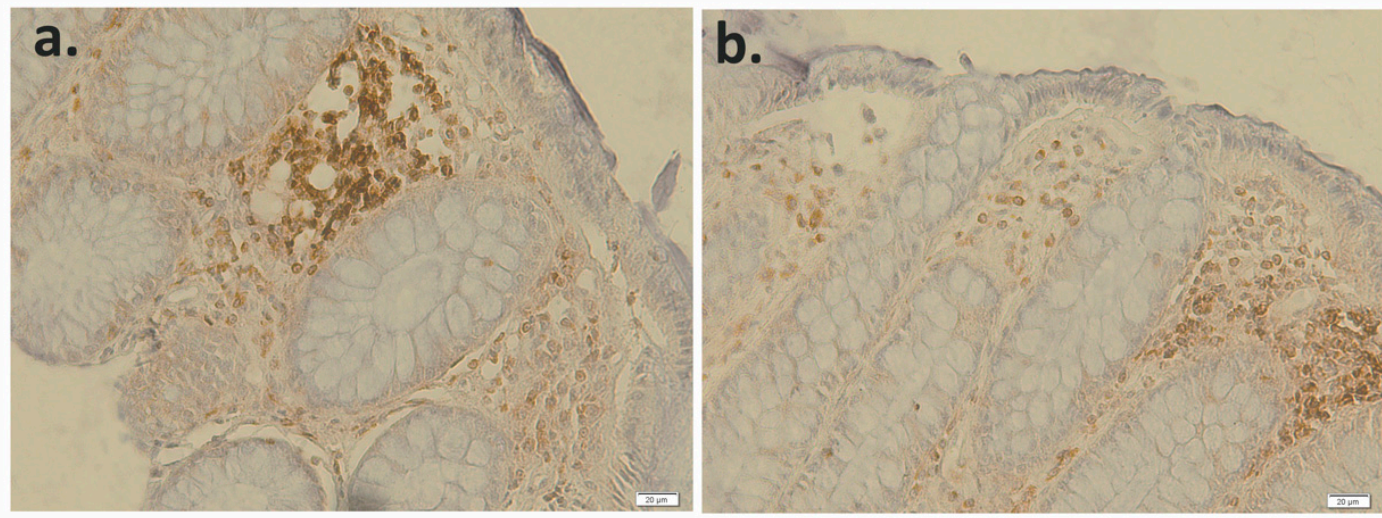

Figure 15. Bcl-2 immunostaining in the proximal (a) and distal (b) sigmoid colon of the onco-hematological patients 3 weeks following epirubicin-based chemotherapy.

The quantification of the immunopositivity showed significant differences in a short time after the epirubicin administration for all markers compared to controls $(p<0.001)$ and almost restored 3 weeks after the chemotherapy cessation (Figure 16).

a.

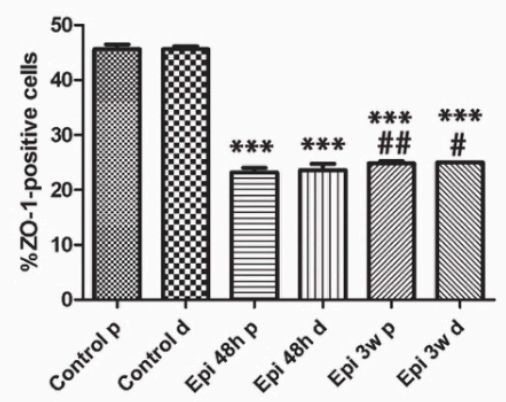

C.

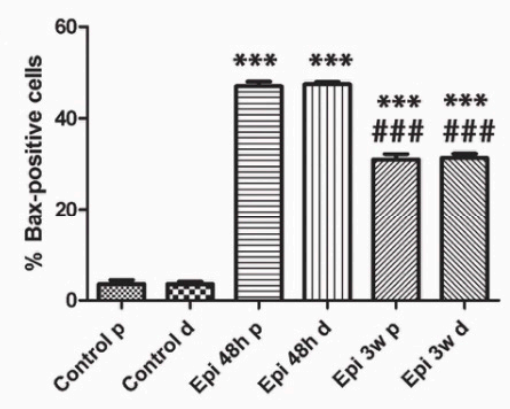

b.

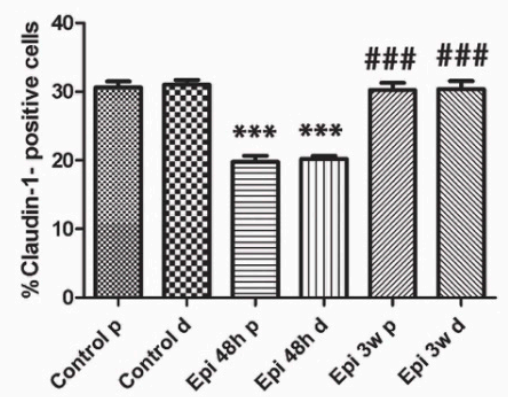

d.

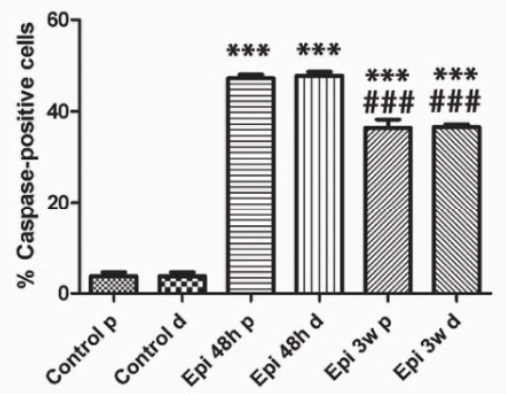

e.

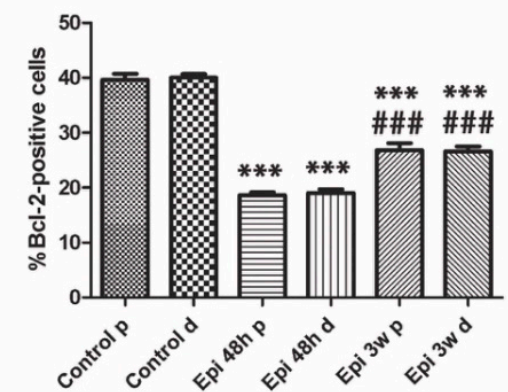

Figure 16. Immunopositivity quantification of ZO-1 (a), Claudin-1 (b), Bax (c), Caspase-3 (d) and $\mathrm{Bcl}-2$ (e) in the proximal (p) and distal (d) sigmoid colon of the onco-hematological patients $48 \mathrm{~h}$ and 3 weeks following epirubicin-based chemotherapy, compared to control. ${ }^{* * *}$ indicates a significant difference at $p<0.001$ vs. control, ${ }^{\# \#}$ significant difference at $p<0.001,{ }^{\#} p<0.01,{ }^{\#} p<0.05$ vs. Epi $48 \mathrm{~h}$. 


\section{Discussion}

Epirubicin is an anthracycline-class antitumor agent that is used in the treatment of several types of cancer, including breast cancer, ovarian, lung cancer and oncohematologic disorders. The antitumor mechanism of epirubicin refers to its ability to intercalate, resulting in a complex formation that interferes with DNA and RNA synthesis [17]. It can also interfere with gene regulation expression by inhibiting polymerase activity [18]. However, it is a nonspecific antitumor drug, and like other chemotherapeutics, causes high DNA damage and selectively targets rapidly proliferating cells such as cancer cells and normal cells with a high self-renewal potential, including those in the gastrointestinal tract [19].

In this respect, we aimed to assess by immunohistochemical assay the consequences of epirubicin-based therapy on the mucosal cells that undergo apoptosis and the injury of the tight junctions, immediately before and after a short time of chemotherapy administration.

The integrity of the intestinal barrier is assured by tight junction, a complex of membrane-bound proteins, occludens and claudins and their junctional adhesion molecule, zonula occludens proteins (ZO)-1, -2 and -3 [20,21]. Recently, clinical and preclinical studies reported altered tight junction protein expression and subsequent altered intestinal permeability in the consecutive administration of Irinotecan or in combination with 5-Fluorouracil [22,23]. Studies have shown a decrease in key tight junction (TJ) proteins, such as ZO-1, in experimental models of intestinal inflammation [24]. Moreover, its depletion corresponds to an increase in intestinal permeability and a decrease in transepithelial resistance $[24,25]$. Our results showed that the expression of essential proteins in the tight junctions, such as Claudin- 1 and ZO-1, is altered $48 \mathrm{~h}$ after epirubicin administration and is not completely restored even 3 weeks after administration, suggesting that the integrity of the intestinal barrier is affected. As a consequence, after chemotherapy, the passage of solutes across the epithelial barrier is impaired due to an increase in paracellular permeability [26].

The Bcl-2 family members are key proteins that regulate the intrinsic apoptotic pathway [27]. Expression of genes that modulate the apoptosis process, such as Bcl-2 (which promotes survival) and Bax (which promote apoptosis), balance cell survival or death after damage [28]. In the present study, we investigate the susceptibility to damage of the intestinal mucosa by epirubicin administration to oncohematological patients. We showed that the expression of the Bcl-2 family (pro-apoptotic Bax and anti-apoptotic Bcl-2) was unbalanced, observing a colonic higher immunopositivity for Bax, detrimental to Bcl-2. Moreover, this finding is in accordance with our preclinical studies, where we found the same pattern in all gastrointestinal tracts of mice [16]. As well, Caspase-3 was highest expressed at $48 \mathrm{~h}$ after epirubicin-based chemotherapy to the oncohematological patients and remain expressed 3 weeks after cessation. Other results show alleviation of epithelial mucositis after administration of NSC321205, the protective effects being mediated by a decrease in Caspase-3 activity and the consequent inhibition of Bax expression [29], which demonstrate that apoptosis is an important event in gastrointestinal mucositis induced by chemotherapy, as well.

\section{Conclusions}

In this study, we assessed the key molecules involved in apoptosis and tight junction intestinal damage and their role in epirubicin-induced intestinal mucositis. The results showed that the Bcl-2 family is involved in intestinal damage via Caspase- 3 dependent apoptosis of epithelial cells. Additionally, the intestinal mucositis activates other injurious pathways through a dramatic drop in Claudin- 1 and ZO-1 expressions, contributing for a while to a structural and functional integrity disruption of the intestinal epithelium.

Author Contributions: Conceptualization, C.C. and A.S.; methodology, C.C., A.S. and E.M.; investigation, A.S., D.I., S.G., A.C., H.H. and E.M.; resources, A.H.; writing-original draft preparation, C.C., A.S. and A.H.; writing-review and editing, C.C., A.H., E.M. and S.G.; supervision, C.C. and A.H. All authors have read and agreed to the published version of the manuscript. 
Funding: This research received no external funding.

Institutional Review Board Statement: The study was conducted according to the guidelines of the Declaration of Helsinki and approved by the Research Ethics Commission of the Arad Clinical Emergency County Hospital (26467/21.09.2016).

Informed Consent Statement: Informed consent was obtained from all subjects involved in the study.

Data Availability Statement: The data presented in this study are available on request from the corresponding author. The data are not publicly available due to privacy.

Conflicts of Interest: The authors declare no conflict of interest.

\section{References}

1. Beck, P.L.; Wong, J.K.; Li, Y.; Swaminathan, S.; Xavier, R.J.; Devaney, K.L.; Podolsky, D.K. Chemotherapy- and RadiotherapyInduced Intestinal Damage Is Regulated by Intestinal Trefoil Factor. Gastroenterology 2004, 126, 796-808. [CrossRef] [PubMed]

2. Kwon, Y. Mechanism-based management for mucositis: Option for treating side effects without compromising the efficacy of cancer therapy. OncoTargets Ther. 2016, 9, 2007-2016. [CrossRef] [PubMed]

3. Birchenough, G.M.; Johansson, M.E.; Gustafsson, J.K.; Bergstrom, J.H.; Hansson, G.C. New developments in goblet cell mucus secretion and function. Mucosal Immunol. 2015, 8, 712-719. [CrossRef] [PubMed]

4. Kim, Y.S.; Ho, S.B. Intestinal goblet cells and mucins in health and disease: Recent insights and progress. Curr. Gastroenterol. Rep. 2010, 12, 319-330. [CrossRef] [PubMed]

5. Rescigno, M. The intestinal epithelial barrier in the control of homeostasis and immunity. Trends Immunol. 2011, 32, 256-264. [CrossRef]

6. Chelakkot, C.; Ghim, J.; Ryu, S.H. Mechanisms regulating intestinal barrier integrity and its pathological implications. Exp. Mol. Med. 2018, 50, 103. [CrossRef]

7. Suzuki, T. Regulation of intestinal epithelial permeability by tight junctions. Cell Mol. Life Sci. 2013, 70, 631-659. [CrossRef]

8. Maloy, K.J.; Powrie, F. Intestinal homeostasis and its breakdown in inflammatory bowel disease. Nature 2011, 474, 298-306. [CrossRef]

9. Khor, B.; Gardet, A.; Xavier, R.J. Genetics and pathogenesis of inflammatory bowel disease. Nature 2011, 474, 307-317. [CrossRef] [PubMed]

10. Feldman, G.J.; Mullin, J.M.; Ryan, M.P. Occludin: Structure, function and regulation. Adv. Drug Deliv. Rev. 2005, 57, 883-917. [CrossRef]

11. Gunzel, D.; Yu, A.S. Claudins and the modulation of tight junction permeability. Physiol. Rev. 2013, 93, 525-569. [CrossRef] [PubMed]

12. Itoh, M.; Furuse, M.; Morita, K.; Saitou, M.; Tsukita, S. Direct binding of three tight junction-associated MAGUKs, ZO-1, ZO-2, and ZO-3, with the COOH termini of claudins. J. Cell Biol. 1999, 147, 1351-1363. [CrossRef]

13. Beatch, M.; Jesaitis, L.A.; Gallin, W.J.; Goodenough, D.A.; Stevenson, B.R. The tight junction protein ZO-2 contains three PDZ (PSD-95/Discs-Large/ZO1) domains and an alternatively spliced region. J. Biol. Chem. 1996, 271, 25723-25726. [CrossRef] [PubMed]

14. Watson, A.J.M.; Pritchard, M.D. Lessons from Genetically Engineered Animal Models VII. Apoptosis in intestinal epithelium: Lessons from transgenic and knockout mice. Am. J. Physiol. Gastrointest. Liver Physiol. 2000, 278, G1-G5. [CrossRef] [PubMed]

15. Hall, P.A.; Coates, P.J.; Ansari, B.; Hopwood, D. Regulation of cell number in the mammalian gastrointestinal tract: The importance of apoptosis. J. Cell Sci. 1994, 107, 3569-3577. [CrossRef]

16. Sasu, A.; Herman, H.; Mariasiu, T.; Rosu, M.; Balta, C.; Anghel, N.; Miutescu, E.; Cotoraci, C.; Hermenean, A. Protective effects of silymarin on epirubicin-Induced mucosal barrier injury of the gastrointestinal tract. Drug Chem. Toxicol. 2015, 38, 442-451. [CrossRef]

17. Cameron, D.; Morden, J.P.; Canney, P.; Velikova, G.; Coleman, R.; Bartlett, J. Accelerated versus standard epirubicin followed by cyclophosphamide, methotrexate, and fluorouracil or capecitabine as adjuvant therapy for breast cancer in the randomised UK TACT2 trial (CRUK/05/19): A multicentre, phase 3, openlabel, randomised, controlled trial. Lancet Oncol. 2017, 18, 929-945.

18. Moretti, E.; Desmedt, C.; Biagioni, C.; Regan, M.M.; Oakman, C.; Larsimont, D.; Galardi, F.; Piccart-Gebhart, M.; Sotiriou, C.; Rimm, D.L. TOP2A protein by quantitative immunofluorescence as a predictor of response to epirubicin in the neoadjuvant treatment of breast cancer. Future Oncol. 2013, 9, 1477-1487. [CrossRef]

19. Yu, J. Intestinal stem cell injury and protection during cancer therapy. Transl. Cancer Res. 2013, 2, 384-396.

20. Will, C.; Fromm, M.; Muller, D. Claudin tight junction proteins: Novel aspects in paracellular transport. Perit. Dial. Int. 2008, 28, 577-584. [CrossRef]

21. Forster, C. Tight junctions and the modulation of barrier function in disease. Histochem. Cell Biol. 2008, 130, 55-70. [CrossRef] [PubMed]

22. Melichar, B.; Dvorak, J.; Krcmova, L.; Hyspler, R.; Urbanek, L.; Solichova, D. Intestinal permeability and vitamin A absorption in patients with chemotherapy-induced diarrhea. Am. J. Clin. Oncol. 2008, 31, 580-584. [CrossRef] [PubMed] 
23. Nakao, T.; Nobuhiro, K.; Komatsu, M.; Yoshikawa, K.; Iwata, T.; Utusnomiya, T.; Shimada, M. Irinotecan injures tight junction and causes bacterial translocation in rat. J. Surg. Res. 2012, 173, 341-347. [CrossRef]

24. Poritz, L.S.; Garver, K.I.; Green, C.; Fitzpatrick, L.; Ruggiero, F.; Koltun, W.A. Loss of the tight junction protein ZO-1 in dextran sulfate sodium induced colitis. J. Surg. Res. 2007, 140, 12-19. [CrossRef] [PubMed]

25. Poritz, L.S.; Harris, L.R.; Kelly, A.A.; Koltun, W.A. Increase in the Tight Junction Protein Claudin-1 in Intestinal Inflammation. Dig. Dis. Sci. 2011, 56, 2802-2809. [CrossRef] [PubMed]

26. Wardill, H.R.; Bowen, J.M.; Gibson, R.J. Chemotherapy-induced gut toxicity: Are alterations to intestinal tight junctions pivotal? Cancer Chemother. Pharmacol. 2012, 70, 627-635. [CrossRef] [PubMed]

27. Leibowitz, B.; Yu, J. Mitochondrial signaling in cell death via the Bcl-2 family. Cancer Biol. Ther. 2010, 9, 417-422. [CrossRef]

28. Pritchard, D.M.; Potten, C.S.; Korsmeyer, S.J.; Roberts, S.; Hickman, J.A. Damage-induced apoptosis in intestinal epithelia from bcl-2-null and bax-null mice: Investigations of the mechanistic determinants of epithelial apoptosis in vivo. Oncogene 1999, 18, 7287-7293. [CrossRef]

29. Talwar, S.; House, R.; Sundaramurthy, S.; Balasubramanian, S.; Yu, H.; Palanisamy, V. Inhibition of caspases protects mice from radiation-induced oral mucositis and abolishes the cleavage of RNA-binding protein HuR. J. Biol. Chem. 2014, 289, 3487-3500. [CrossRef] 